\title{
Can we obtain in vivo transmural mean hoop stress of the aortic wall without knowing patient-specific material properties and residual deformations?
}

\author{
Minliang Liu ${ }^{1}$, Liang Liang ${ }^{1}$, Haofei Liu², Ming Zhang ${ }^{2}$, Caitlin Martin ${ }^{1}$, Wei Sun ${ }^{1}$ \\ ${ }^{1}$ Tissue Mechanics Laboratory \\ The Wallace H. Coulter Department of Biomedical Engineering \\ Georgia Institute of Technology and Emory University, Atlanta, GA \\ ${ }^{2}$ Department of Mechanics, Tianjin University \\ 92 Weijin Road, Tianjin, China 300072
}

Submitted to

Some Journal

$7 / 10 / 2018$

\begin{abstract}
For correspondence:
\end{abstract}
Wei Sun, Ph.D.

The Wallace H. Coulter Department of Biomedical Engineering

Georgia Institute of Technology and Emory University

Technology Enterprise Park, Room 206

387 Technology Circle, Atlanta, GA 30313-2412

Tel:(404) 385-1245; Email: wei.sun@bme.gatech.edu 


\section{Abstract}

33 It is well known that residual deformations/stresses alter the mechanical behavior of arteries, e.g.

34 the pressure-diameter curves. In an effort to enable personalized analysis of the aortic wall stress,

35 approaches have been developed to incorporate experimentally-derived residual deformations into

36 in vivo loaded geometries in finite element simulations using thick-walled models. Solid elements

37 are typically used to account for "bending-like” residual deformations. Yet, the difficulty in

38 obtaining patient-specific residual deformations and material properties has become one of the

39 biggest challenges of these thick-walled models. In thin-walled models, fortunately, static

40 determinacy offers an appealing prospect that allows for the calculation of the thin-walled

41 membrane stress without patient-specific material properties. The membrane stress can be

42 computed using forward analysis by enforcing an extremely stiff material property as penalty

43 treatment, which is referred to as the forward penalty approach. However, thin-walled membrane

44 elements, which have zero bending stiffness, are incompatible with the residual deformations, and

45 therefore, it is often stated as a limitation of thin-walled models. In this paper, by comparing the

46 predicted stresses from thin-walled models and thick-walled models, we demonstrate that the

47 transmural mean hoop stress is the same for the two models and can be readily obtained from in

48 vivo clinical images without knowing the patient-specific material properties and residual

49 deformations. Computation of patient-specific mean hoop stress can be greatly simplified by using

50 membrane model and the forward penalty approach, which may be clinically valuable.

51 Keywords: residual stress; transmural mean hoop stress; membrane stress; forward penalty 52 approach. 


\section{Introduction}

Residual deformations/stresses first discovered in the 1980s (Chuong and Fung 1986;

Vaishnav and Vossoughi 1983) have been shown to significantly affect the physiological wall stress distributions (Delfino et al. 1997; Fung 1991; Holzapfel et al. 2000; Humphrey 2002; Matsumoto and Hayashi 1996). To incorporate residual deformations in arteries, traditional forward analysis uses a thick-walled model starting from the stress-free reference configuration.

59 Then deformation relations, constitutive laws and equilibrium equations are utilized to solve the boundary value problem. However, when applying this conventional approach to obtain patientspecific stress fields from the in vivo loaded geometries in clinical images, one has to first determine the unknown material parameters and residual deformations, which are required in the thick-walled finite element (FE) models. Some studies have suggested the use of experimentallydetermined material and residual deformation parameters (Alastrué et al. 2010; Pierce et al. 2015). However, using residual deformations and material properties that are not patient-specific is a clear limitation.

Fortunately, for a specific type of biological membrane structures such as the aorta, the wall stress is nearly insensitive to the variation of material properties. This property is called static determinacy, i.e. the external force (pressure) along the geometry can be used to directly compute the internal tension/stress. This is because the vessel wall can be seen as locally in a plane stress state (Miller and Lu 2013), the solution of the equilibrium is weakly sensitive to the material

72 properties. The aorta is shown to be approximately statically determinate (Joldes et al. 2016; Liu

73 et al. 2017). Thus, its stress distribution can be directly obtained using membrane elements by a

74 forward penalty method (Joldes et al. 2016; Lu and Luo 2016) which enforces an extremely stiff 75 material property as penalty treatment. The computation of the thin-walled stress can be greatly 
simplified by this forward approach. However, due to the assumption of no bending stiffness in

77 the membrane elements, the self-equilibrium residual deformations are inadmissible to the thinwalled models, which is often stated as a limitation of such models.

In this paper, by comparing the predicted stresses from thin-walled models with thickwalled models considering residual deformations, we demonstrate that the transmural mean hoop

81 stress (i.e., averaged stress through the thickness) fields are the same for the two models. Thus, the

82 transmural mean hoop stress can be readily obtained from in vivo clinical images using the forward

83 penalty approach without knowing the patient-specific material properties and residual 84 deformations. The remaining sections are organized as follows. In Section 2, the theoretical 85 arguments are described and the validity is shown by analytical examples. Thin-walled and thickwalled FE models with a patient-specific geometry are demonstrated in Section 3. In Section 4,

87 the discussion and conclusions are presented.

\section{Theoretical and Analytical Arguments} hoop stress by assuming a perfect cylindrical shape of the aorta.

$$
\sigma_{\theta \theta}=\frac{P a}{t}
$$

92 where $\sigma_{\theta \theta}$ is the hoop stress in the thin-walled tube, $P$ is the pressure, $a$ is the inner radius, and $t$

93 is the in vivo wall thickness. The material properties are not involved in this equation, and stress

94 is directly calculated using the static force equilibrium. Opposite to the middle radius value used

95 in (Horný et al. 2014), we emphasize that inner radius should be used as the blood pressure is applied to the inner surface of the aorta. 
It is well known that residual stresses alter the mechanical response of arteries, e.g. the pressure-diameter curves (Holzapfel et al. 2000). Nonetheless, from the static determinacy prospective, for the in vivo loaded configuration, the equilibrium between the resultant force and the external pressure load should always hold, and thus, the hoop stress resultant (tension) should be insensitive to the material parameters and residual deformations. This implies that no matter how the aorta is internally balanced or residually stressed, the wall tension can always be computed only using the static equilibrium. Therefore, when the wall thickness is given, the simple thinwalled model would be sufficient in determining the transmural mean hoop stress.

\subsection{The Opening Angle Method}

In this subsection, we use an analytical example to demonstrate that the mean hoop stress is insensitive to the change of opening angles. We assume that the residual stress can be described by the opening angle and that the aorta can be modelled as a perfect tube. and (Pierce et al. 2015), the total deformation gradient tensor of the tube taking into account the

111 residual stress, $\boldsymbol{F}_{\text {total }}$, can be obtained as

$$
\boldsymbol{F}_{\text {total }}(r)=\frac{r k}{\left[A^{2}+k \frac{l}{L}\left(r^{2}-a^{2}\right)\right]^{\frac{1}{2}}} \boldsymbol{e}_{\theta} \otimes \boldsymbol{E}_{\Theta}+\frac{l}{L} \boldsymbol{e}_{Z} \otimes \boldsymbol{E}_{Z}+\frac{L}{r k l}\left[A^{2}+k \frac{l}{L}\left(r^{2}-a^{2}\right)\right]^{\frac{1}{2}} \boldsymbol{e}_{r} \otimes \boldsymbol{E}_{R}
$$

113 where $r \in[a, b], a$ and $b$ are the inner and outer radii of the in vivo deformed geometry. $k$,

114 defined as $k=\frac{2 \pi}{2 \pi-\alpha}$, is used to describe the opening angle $\alpha$. $A$ and $B$ are the inner and outer radii 115 of the stress-free geometry. $L$ and $l$ are the axial length of the aorta segment in the stress-free and deformed geometry, respectively. $\boldsymbol{E}_{\Theta}, \boldsymbol{E}_{Z}$ and $\boldsymbol{E}_{R}$ and $\boldsymbol{e}_{\theta}, \boldsymbol{e}_{z}$ and $\boldsymbol{e}_{r}$ are the unit basis vectors for 
117 the stress-free and deformed geometry respectively. To make the solution simple, the constitutive

118 relation of the aorta tissue is modelled using the isotropic Neo-Hookean model. The strain energy

$119 \Psi$ is

$$
\Psi=\frac{1}{2} \mu\left(I_{1}-3\right)
$$

121

122

123

124

125

126

127

128

129

130

131

132

133

134

135

136

where $\mu$ is the shear modulus and $I_{1}$ is the first invariant. To solve for the in vivo stress when systolic blood pressure ( $P=104 \mathrm{mmHg}$ ) (Martin et al. 2015) is present, we utilize the stress equilibrium equation, which can be expressed in the radial equation

$$
\frac{d \sigma_{r r}}{d r}=\frac{1}{r}\left(\sigma_{r r}-\sigma_{\theta \theta}\right)
$$

where $\sigma_{\theta \theta}$ and $\sigma_{r r}$ are the stresses in the circumferential and radial direction respectively. Eqn. (4)

can be reduced to $\frac{d \sigma_{r r}}{d r}=\frac{\mu}{r}\left(\lambda_{\theta}^{2}-\lambda_{r}^{2}\right)$ (Holzapfel and Ogden 2010), with $\lambda_{\theta}$ and $\lambda_{r}$ referring to the stretches in the circumferential and radial directions, respectively. By solving the equilibrium Eqn. (4), together with the traction continuity condition $\sigma_{r r}(a)=-P$, we are able to obtain the radial stress (Holzapfel and Ogden 2010)

$$
\sigma_{r r}(r)=-P+\mu\left[\frac{k}{\lambda_{z}} \log \left(\frac{\lambda_{r} \lambda_{z} r k}{A}\right)-\frac{1}{k \lambda_{z}} \log \left(\frac{r}{a}\right)+\frac{1}{2}\left(\lambda_{r}^{2}-\frac{1}{k \lambda_{z}}\right)\left(\frac{a^{2}-r^{2}}{a^{2}}\right)\right]
$$

where $\lambda_{z}$ is the stretch in the axial direction. The hoop stress is then calculated using

$$
\sigma_{\theta \theta}(r)=\sigma_{r r}(r)+\mu\left(\lambda_{\theta}^{2}-\lambda_{r}^{2}\right)
$$

The geometry of the aorta in clinical images is always in the in vivo deformed state, from which the opening angle is not measurable. To this end, we fixed the inner and outer radii of the in vivo deformed geometry, $a$ and $b$, for all scenarios and vary the opening angle from 0 to 330 degree.

For a certain opening angle $\alpha$, the inner and outer radii $A$ and $B$ of the cut-open sectors are 
137 solved using the boundary condition $\sigma_{r r}(b)=0$ and the assumption of incompressibility $b^{2}=$

$138 a^{2}+\frac{1}{k \lambda_{z}}\left(B^{2}-A^{2}\right)$. Related parameters are listed in Table 1 and values of $A$ and $B$ are shown in

139 Table 2. The transmural mean hoop stress is defined as

$$
\bar{\sigma}_{\theta \theta}=\frac{1}{b-a} \int_{a}^{b} \sigma_{\theta \theta} d r
$$
exactly the same as the thin-walled hoop stress calculated using Eqn. (1). Unsurprisingly, if we

rewrite Eqn. (4) as $\sigma_{\theta \theta}=\frac{d}{d r}\left(r \sigma_{r r}\right)$ and therefore $\bar{\sigma}_{\theta \theta}=\frac{1}{b-a} \int_{a}^{b} d\left(r \sigma_{r r}\right)=\frac{b \sigma_{r r}(b)-a \sigma_{r r}(a)}{t}=\frac{P a}{t}$,

which is exactly the same formula as the Laplace law. In addition, as shown in Figure 1 (right),

Section 3.2.1, parameters shown in Table 3) would not affect the static determinacy. The inner hoop stress tends to be reduced while the outer hoop stress is increased when gradually increasing 2015), they are presented here for illustrative purpose.

\subsection{The Layer-Specific Three-Dimensional Residual Stress Model}


157 applied to the residually-stressed aorta. The result indicates that the transmural mean hoop stress

158 is independent of residual deformations.

$\boldsymbol{F}_{R S}^{(I)}\left(r^{(I)}\right)=\frac{r^{(I)} k^{(I)}}{\left[A^{(I)^{2}}+k^{(I)} \frac{l}{L^{(I)}}\left(r^{(I)^{2}}-a^{(I)}\right)\right]^{\frac{1}{2}}} \boldsymbol{e}_{\theta} \otimes \boldsymbol{E}_{\Theta}+\frac{l}{L^{(I)}} \boldsymbol{e}_{Z} \otimes \boldsymbol{E}_{Z}+\frac{L^{(I)}}{r^{(I)} k^{(I)} l}\left[A^{(I)^{2}}+k^{(I)} \frac{l}{L^{(I)}}\left(r^{(I)^{2}}-\right.\right.$

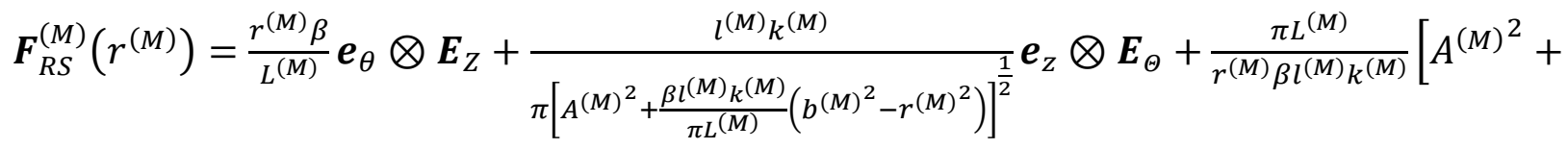

$\left.164 \quad \frac{\beta l^{(M)} k^{(M)}}{\pi L^{(M)}}\left(b^{(M)^{2}}-r^{(M)^{2}}\right)\right]^{\frac{1}{2}} \boldsymbol{e}_{r} \otimes \boldsymbol{E}_{R}$

$\boldsymbol{F}_{R S}^{(A)}\left(r^{(A)}\right)=\frac{\pi r^{(A)}}{L_{2}^{(A)}} \boldsymbol{e}_{\theta} \otimes \boldsymbol{E}_{X_{2}}+\frac{l}{L_{3}^{(A)}} \boldsymbol{e}_{Z} \otimes \boldsymbol{E}_{X_{3}}+\frac{L_{2}^{(A)} L_{3}^{(A)}}{\pi r^{(A)} l} \boldsymbol{e}_{r} \otimes \boldsymbol{E}_{X_{1}}$ of the related parameters are listed in Table 4.

171 inner surface of the aorta, and we assume no axial tension caused by in vivo loading conditions.

172 The residual axial stretches have been incorporated in the deformation gradient tensors of each

173 layer. The transmural mean hoop stress for the three layer composite is defined as

$$
\bar{\sigma}_{\theta \theta}=\frac{1}{b^{(A)}-a^{(I)}} \sum_{i=I, M, A} \int_{a^{(i)}}^{b^{(i)}} \sigma_{\theta \theta}^{(i)} d r
$$


175 As depicted in Figure 2, the mean hoop stress is identical to the thin-walled hoop stress.

176

\section{Finite Element Analyses Incorporating Residual Deformations}

In this section, irregularity of patient-specific geometries are taken into account using FE analyses. The validity of the conclusion in Section 2 is examined by a real patient geometry. The forward penalty approach (Section 3.1) is used to estimate the thin-walled membrane stress. For the thick-walled FE models, the generalized pre-stressing algorithm (GPA) (Pierce et al. 2015; Weisbecker et al. 2014) is implemented in ABAQUS (Section 3.2) to predict the in vivo stress distribution with both the residual deformations and the pre-stresses incorporated.

A CT-derived geometry from the ascending thoracic aortic aneurysm (ATAA) patient (Martin et al. 2015) were used. The inner surface of the aortic wall was divided into 4,950 M3D4 membrane elements in ABAQUS, using the automatic algorithm (Liang et al. 2017) previously developed by our group. Mesh sensitivity analysis was performed in our previous work (Martin et al. 2015). Due to partial volume effect, the wall thickness is difficult to infer from CT images, therefore a constant deformed thickness of $1.5 \mathrm{~mm}$ was assumed based on (Liang et al. 2017). Sensitivity analyses with respect to the wall thickness were carried out in Section 3.2.2. Next, the membrane mesh was extruded outwardly to create two solid meshes (C3D8 elements) with 8 and 9 layers in Section 3.2.2 and Section 3.2.3, respectively.

\subsection{A Thin-walled Model using the Forward Penalty Approach}

The prediction of the in vivo stress of the aortic wall has been relied on the recovery of the unloaded state and the incorporation of residual deformations, which requires the use of iterative techniques (Alastrué et al. 2010). A simple and effective forward penalty approach (Joldes et al. 2016) (Lu 
and Luo 2016) has been recently proposed to predict the in vivo membrane stress without knowing the material properties. In statically determinant structures, the stress is independent of the material properties, it would be legitimate to assume an extremely stiff property, so that the deformation/change of shape from the unloaded configuration to the loaded configuration is infinitesimal/negligible. This allows us to use the in vivo configuration as the unloaded configuration because the deformation is infinitesimal. In the forward method, an artificially stiff material property (i.e. $\mu=10^{7} \mathrm{~Pa}$, Neo-Hookean model) is assigned to the aortic wall, realizing a penalty treatment to enforce a nearly rigid condition (Lu and Luo 2016). When the in vivo pressure is applied to the in vivo, image-derived geometry, the deformation would be infinitesimal due to the high stiffness of the material. The correct in vivo membrane stress field is readily obtained in this forward analysis due to the fact that the aortic wall is approximately statically determinate. This approach was shown as effective as iterative approach (Lu and Luo 2016).

Similar to the reason for the use of the inner radius in the Laplace equation (Eqn. (1)), we applying the forward approach.

$$
\Psi=C_{10}\left(\overline{\boldsymbol{I}}_{1}-3\right)+\frac{k_{1}}{2 k_{2}} \sum_{i=1}^{2}\left[\exp \left\{k_{2}\left[\kappa \overline{\boldsymbol{I}}_{1}+(1-3 \kappa) \overline{\boldsymbol{I}}_{4(6)}-1\right]^{2}\right\}-1\right]+\frac{1}{D}\left[\frac{J^{2}-1}{2}-\ln J\right]
$$


217 where $C_{10}, k_{1}, k_{2}$ and $\kappa$ are material parameters, $\theta$ defines the fiber directions, please refer to

218 (Abaqus 2014; Gasser et al. 2006) for detailed definitions. The parameter $D$ enforces the nearly

219 incompressibility and is fixed to be $1 \times 10^{-5}$.

The GPA (Pierce et al. 2015; Weisbecker et al. 2014) is utilized to incorporate the residual

221 deformation. The total deformation gradient $\boldsymbol{F}_{t}$ is stored as a history variable for each integration

222 point. $\boldsymbol{F}_{t}$ is updated based on the incremental deformation gradient $\Delta \boldsymbol{F}$ resulting from the

223 prescribed load and boundary conditions.

$$
\boldsymbol{F}_{t+1}=\Delta \boldsymbol{F} \boldsymbol{F}_{t}
$$

The incremental deformation gradient of the residual stress $\Delta \boldsymbol{F}_{R S}$ is first iteratively applied to the

226 image-derived geometry and stored in $\boldsymbol{F}_{t}$. Next, the incremental deformation gradient of the pre-

227 stress $\Delta \boldsymbol{F}_{P S}$ resulting from the in vivo blood pressure is incrementally applied and stored in $\boldsymbol{F}_{t}$.

228 Thus, deformation gradient tensors associated with the residual stress $\boldsymbol{F}_{R S}$ and the pre-stress $\boldsymbol{F}_{P S}$

229 are accounted sequentially. The GPA is implemented in the ABAQUS user subroutine UMAT.

230 The implementation was validated by comparing the analytical and FE results as in (Pierce et al.

231 2015).

\subsubsection{Thick-walled Models with Various Opening Angles}

The thick-walled solid elements were utilized in this section to encompass the opening

234 angle. Various values of the opening angle were incorporated through the GPA. Small opening

235 angles may be unusual to observe in experiments, they are shown here for illustration purposes.

236 The aorta was modelled as a single layer wall. This assumption may be relevant to abdominal

237 aneurysmal tissue since collagen structure becomes nearly homogenous across the entire wall 
238 (Gasser et al. 2012). For ascending aortic aneurysms, collagen organization may be different in 239 different layers (Sassani et al. 2015). The GOH model (Eqn (10)) was used as the constitutive law, 240 and the material parameters (shown in Table 3) were determined from fitting the biaxial data from 241 (Martin et al. 2015) of the particular patient. Mean absolute percentage error (MAPE) was used to compare the transmural mean hoop stress (Eqn.(7)) of the thin-walled and thick-walled models:

$$
M A P E=\frac{1}{N} \sum_{i=1}^{N}\left|\frac{\bar{\sigma}_{\theta \theta, i}{ }^{(t h i n)}-\bar{\sigma}_{\theta \theta, i}{ }^{(t h i c k)}}{\bar{\sigma}_{\theta \theta, i}{ }^{(t h i n)}}\right|
$$

245 where $\bar{\sigma}_{\theta \theta, i}{ }^{(\text {thin })}$ and $\bar{\sigma}_{\theta \theta, i}{ }^{(\text {thick })}$ are the transmural mean hoop stress predicted by the thin-

246 walled and thick-walled models respectively. $i$ is an element index for the thin-walled model and $247 \quad N$ is the number of elements.

To study the sensitivity of the MAPE of the transmural mean stress with respect to the 249 thickness, three representative thickness values (1mm, $2 \mathrm{~mm}$ and $3 \mathrm{~mm}$ ) were chosen with $\alpha=$ $250120^{\circ}$. The results are summarized in Table 5 . Note that this opening angle value is chosen because 251 the corresponding stress distribution is close to homogenized state in the FE simulation, and this 252 value may not be consistent with the average value obtained from experiment (Sokolis 2015). We 253 also notice that opening angle values are widely distributed according to (Sokolis 2015), 120 254 degree can be considered as a feasible value.

$$
\operatorname{STPE}(i)=\operatorname{sign}\left[\sigma_{\theta \theta, i}{ }^{(\text {thick })}\left(a_{i}\right)-\sigma_{\theta \theta, i}{ }^{(\text {thick })}\left(b_{i}\right)\right] \frac{1}{b_{i}-a_{i}} \int_{a_{i}}^{b_{i}}\left|\frac{\sigma_{\theta \theta, i}{ }^{(t h i c k)}(r)-\bar{\sigma}_{\theta \theta, i}{ }^{(t h i c k)}}{\bar{\sigma}_{\theta \theta, i}{ }^{(t h i c k)}}\right| d r
$$


where $a_{i}$ and $b_{i}$ represent the inner and outer radii respectively, and $r$ is the radius. The sign is given based on the difference between the inner and outer wall hoop stress. If the inner wall stress is greater than the outer, the STPE is positive, otherwise the STPE is negative.

The results are shown in Figure 3, the transmural mean hoop stress fields are almost identical for various opening angles and the forward penalty approach. More detailed views of ring cuts at the same location are shown in Figure 4. With increased opening angle, the mean signed transmural percentage error (MSTPE) changes from positive to negative.

The probability density functions (PDFs) of the STPE are plotted in Figure 5. The PDFs are fitted using the Gaussian distribution. It can be observed that the PDF shifts leftward with the increase of the opening angle.

\subsubsection{A Thick-walled Model with Layer-Specific Three-Dimensional}

\section{Residual Deformation}

In this section, the deformation gradient tensors $\boldsymbol{F}_{R S}^{(i)}(i=I, M, A)$ of Section 2.2 was incorporated in a FE simulation using the GPA. The ratio of intima, media and adventitia (18.53\%, 45.56\%, 35.91\%) and the layer-specific GOH parameters (shown in Table 6) were taken from the median experimental value for human thoracic aortas in (Weisbecker et al. 2012). Layer-specific material parameter data for ATAA is also available in (Sassani et al. 2015; Sokolis et al. 2012). The geometrical parameters determining the residual deformation of abdominal aorta from (Holzapfel and Ogden 2010), same as Section 2.2 (Table 4), were directly used for the ATAA patient. (Sokolis 2015) documented layer-specific residual stretch and opening angle data for ATAA. Unfortunately, it is not compatible with the current three-dimensional residual stress model 
279 (Holzapfel and Ogden 2010). Specifically, (Holzapfel and Ogden 2010) considered different 280 geometries of reference configurations for different layers and would need more complicated 281 experimental setups. the transmural mean stress field predicted by the forward penalty approach and the GPA are, again, almost identical, with a MAPE of 3.98\% (Figure 6, second row). Since the details of transmural hoop stress distributions in a ring predicted by method described in Section 3.1 (forward, membrane), Section 3.2.2 (opening angle $\alpha=180^{\circ}$ ) and Section 3.2.3 (layer-specific 3D residual deformation), respectively.

\section{Discussions and Conclusions}

One of the biggest obstacles in the field of biomechanical analysis of the aorta is the difficulty in obtaining both the patient-specific material properties and the patient-specific residual deformations from in vivo clinical images. This paper offers an appealing prospect that the mean

293 hoop stress (or hoop wall tension) of the aortic wall can be computed without knowing the mechanical properties and the residual deformations of the aortic tissue. Computation of patient-

295 specific mean hoop stress can be greatly simplified by using membrane model and the forward penalty approach, which may be clinically valuable. In some wall strength tests (Ferrara et al. 2016; Pham et al. 2013), the intact wall is tested without separation of each individual layer, which corresponds to the averaged wall strength across the wall thickness, consistent with the membrane 
to calculate an approximation of rupture risk such as the rupture potential index (RPI) (Vande Geest et al. 2006).

Because of the difference in constituents and thus mechanical properties, the hoop stress distribution may not be uniform in multi-layer models. The iterative approaches such as the GPA, may yield detailed results with through-thickness and layer-specific stress distributions using multilayered thick-walled models. Therefore, it would be natural to combine layer-specific wall stress distribution with available layer-specific wall strength data (Sokolis et al. 2012) for a more detailed rupture/dissection analysis. Nonetheless, residual deformations are shown to be highly patient-specific and axial location-dependent (Sokolis 2015). Elastic properties also exhibit regional (Iliopoulos et al. 2009; Sassani et al. 2015) and intra-patient (Martin et al. 2015) variations. Thus, such complex patient- and layer-specific residual deformation and elastic property fields need to be noninvasively estimated for an accurate modeling prediction of clinical events (e.g. rupture). Currently, it is impossible to estimate the layer-specific and heterogeneous material and residual deformation parameters simultaneously from in vivo clinical images. We admit that hoop stress within each layer may be more useful than mean hoop stress for predicting some clinical adverse events such as aortic dissection. However, the mean hoop stress is clinically valuable too because it is patient-specific, which does not depend on material parameters and residual deformations.

The inclusion of residual deformation often reduces the hoop stress gradient, and thus tends to homogenize the hoop stress distribution in the in vivo deformed configuration (Chaudhry et al. 1997; Chuong and Fung 1986; Fung 1991; Holzapfel et al. 2000; Humphrey 2002; Raghavan et al. 2004). This makes the thin-walled hoop stress, or the mean hoop stress more physiologically relevant in the sense that it represents the ideal homogenized wall stress in single layer models. 
323 Homogenized stress state is an assumption for some growth models, e.g., (Polzer et al. 2013), and

324 the method proposed in (Schröder and Brinkhues 2014) is based on smoothing the stress gradient.

325 In this study, the incorporation of opening angles also tends to homogenize the hoop stress

326 distribution as shown in Figure 1. In Figure 3, the MSTPE is close to 0 when 120 180 degree

327 opening angle is incorporated. However, this value seems to be lower than the average value

328 obtained from experiment (Sokolis 2015). This might be due to the assumption of uniform material 329 properties and uniform thickness in the computational model, which could impact the transmural 330 stress distribution. We also notice that a wide range of opening angle is documented in (Sokolis 331 2015), 120 180 degree opening angle can be considered feasible.

The transmural mean axial/longitudinal stress of the aorta may be statically determinant 333 when the longitudinal force is known. The ascending aorta also has in vivo longitudinal 334 deformations/stretches due to the heart movements during cardiac cycles. Such boundary condition 335 is very complex and it can be difficult to model in a FE simulation. In the present study, a simplified 336 boundary condition was used: the boundary nodes were only allowed to move in the radial 337 directions. We have tried different boundary conditions such as prescribing the longitudinal forces, 338 but encountered convergence problems in the FE simulations. The in vivo longitudinal boundary 339 conditions would significantly impact the longitudinal stress field, which warrants further studies 340 in the future.

In conclusion, due to static determinacy, the transmural mean hoop stress in the in vivo 342 configuration of the aorta is independent of mechanical properties and residual deformations. The 343 forward penalty method, which enforces a rigid condition as the penalty treatment, can greatly 344 simplify the computation of the mean hoop stress for patient-specific geometries. 


\section{Acknowledgements}

This study is supported in part by NIH grants HL104080 and HL127570. Liang is

supported by an American Heart Association postdoctoral fellowship 16POST30210003.

\section{Conflict of Interest}

The authors declare that they have no conflict of interest.

\section{References}

Abaqus (2014) Abaqus 6.14 Documentation.

Alastrué V, Garía A, Peña E, Rodríguez JF, Martínez MA, Doblaré M (2010) Numerical framework for patient-specific computational modelling of vascular tissue International Journal for Numerical Methods in Biomedical Engineering 26:35-51 doi:10.1002/cnm.1234

Chaudhry HR, Bukiet B, Davis A, Ritter AB, Findley T (1997) Residual stresses in oscillating thoracic arteries reduce circumferential stresses and stress gradients Journal of Biomechanics 30:57-62 doi:http://dx.doi.org/10.1016/S0021-9290(97)81292-4

Chuong CJ, Fung YC (1986) Residual Stress in Arteries. In: Schmid-Schönbein GW, Woo SLY, Zweifach BW (eds) Frontiers in Biomechanics. Springer New York, New York, NY, pp 117-129. doi:10.1007/978-1-4612-4866-8_9

Delfino A, Stergiopulos N, Moore JE, Meister JJ (1997) Residual strain effects on the stress field in a thick wall finite element model of the human carotid bifurcation Journal of Biomechanics 30:777-786 doi:http://dx.doi.org/10.1016/S0021-9290(97)00025-0

Ferrara A, Morganti S, Totaro P, Mazzola A, Auricchio F (2016) Human dilated ascending aorta: Mechanical characterization via uniaxial tensile tests Journal of the Mechanical Behavior of Biomedical Materials 53:257-271 doi:http://dx.doi.org/10.1016/i.jmbbm.2015.08.021

Fung YC (1991) What are the residual stresses doing in our blood vessels? Annals of Biomedical Engineering 19:237-249 doi:10.1007/bf02584301

Gasser TC, Gallinetti S, Xing X, Forsell C, Swedenborg J, Roy J (2012) Spatial orientation of collagen fibers in the abdominal aortic aneurysm's wall and its relation to wall mechanics Acta Biomaterialia 8:3091-3103 doi:http://dx.doi.org/10.1016/j.actbio.2012.04.044

Gasser TC, Ogden RW, Holzapfel GA (2006) Hyperelastic modelling of arterial layers with distributed collagen fibre orientations Journal of The Royal Society Interface 3:15-35 doi:10.1098/rsif.2005.0073

Guo X, Kassab GS (2003) Variation of mechanical properties along the length of the aorta in C57bl/6 mice American Journal of Physiology - Heart and Circulatory Physiology 285:H2614-H2622 doi:10.1152/ajpheart.00567.2003

Holzapfel GA, Gasser TC, Ogden RW (2000) A New Constitutive Framework for Arterial Wall Mechanics and a Comparative Study of Material Models Journal of elasticity and the physical science of solids 61:1-48 doi:10.1023/a:1010835316564

Holzapfel GA, Ogden RW (2010) Modelling the layer-specific three-dimensional residual stresses in arteries, with an application to the human aorta Journal of The Royal Society Interface 7:787799 doi:10.1098/rsif.2009.0357 
Holzapfel GA, Sommer G, Auer M, Regitnig P, Ogden RW (2007) Layer-Specific 3D Residual Deformations of Human Aortas with Non-Atherosclerotic Intimal Thickening Annals of Biomedical Engineering 35:530-545 doi:10.1007/s10439-006-9252-z

Horný L, Netušil M, Voňavková T (2014) Axial prestretch and circumferential distensibility in biomechanics of abdominal aorta Biomechanics and Modeling in Mechanobiology 13:783-799 doi:10.1007/s10237-013-0534-8

Humphrey JD (2002) Cardiovascular solid mechanics. Cells, tissues, and organs doi:10.1016/S00219290(03)00032-0

Iliopoulos DC et al. (2009) Regional and directional variations in the mechanical properties of ascending thoracic aortic aneurysms Medical Engineering \& Physics 31:1-9 doi:http://dx.doi.org/10.1016/j.medengphy.2008.03.002

Joldes GR, Miller K, Wittek A, Doyle B (2016) A simple, effective and clinically applicable method to compute abdominal aortic aneurysm wall stress Journal of the Mechanical Behavior of Biomedical Materials 58:139-148 doi:https://doi.org/10.1016/i.jmbbm.2015.07.029

Liang L, Liu M, Martin C, Elefteriades JA, Sun W (2017) A machine learning approach to investigate the relationship between shape features and numerically predicted risk of ascending aortic aneurysm Biomechanics and Modeling in Mechanobiology doi:10.1007/s10237-017-0903-9

Liu M, Liang L, Sun W (2017) A new inverse method for estimation of in vivo mechanical properties of the aortic wall Journal of the Mechanical Behavior of Biomedical Materials 72:148-158 doi:http://dx.doi.org/10.1016/i.jmbbm.2017.05.001

Lu J, Luo Y (2016) Solving membrane stress on deformed configuration using inverse elastostatic and forward penalty methods Computer Methods in Applied Mechanics and Engineering 308:134150 doi:http://dx.doi.org/10.1016/j.cma.2016.05.017

Martin C, Sun W, Elefteriades J (2015) Patient-specific finite element analysis of ascending aorta aneurysms American Journal of Physiology - Heart and Circulatory Physiology 308:H1306-H1316 doi:10.1152/ajpheart.00908.2014

Matsumoto T, Hayashi K (1996) Stress and Strain Distribution in Hypertensive and Normotensive Rat Aorta Considering Residual Strain Journal of Biomechanical Engineering 118:62-73 doi:10.1115/1.2795947

Miller K, Lu J (2013) On the prospect of patient-specific biomechanics without patient-specific properties of tissues Journal of the Mechanical Behavior of Biomedical Materials 27:154-166 doi:http://dx.doi.org/10.1016/i.jmbbm.2013.01.013

Pham T, Martin C, Elefteriades J, Sun W (2013) Biomechanical characterization of ascending aortic aneurysm with concomitant bicuspid aortic valve and bovine aortic arch Acta Biomaterialia 9:7927-7936 doi:http://dx.doi.org/10.1016/i.actbio.2013.04.021

Pierce DM et al. (2015) A method for incorporating three-dimensional residual stretches/stresses into patient-specific finite element simulations of arteries Journal of the Mechanical Behavior of Biomedical Materials 47:147-164 doi:http://dx.doi.org/10.1016/j.jmbbm.2015.03.024

Polzer S, Bursa J, Gasser TC, Staffa R, Vlachovsky R (2013) A Numerical Implementation to Predict Residual Strains from the Homogeneous Stress Hypothesis with Application to Abdominal Aortic Aneurysms Annals of Biomedical Engineering 41:1516-1527 doi:10.1007/s10439-013-0749-y

Raghavan ML, Trivedi S, Nagaraj A, McPherson DD, Chandran KB (2004) Three-Dimensional Finite Element Analysis of Residual Stress in Arteries Annals of Biomedical Engineering 32:257-263 doi:10.1023/b:abme.0000012745.05794.32

Sassani SG, Tsangaris S, Sokolis DP (2015) Layer- and region-specific material characterization of ascending thoracic aortic aneurysms by microstructure-based models Journal of Biomechanics 48:3757-3765 doi:10.1016/j.jbiomech.2015.08.028 
Schröder J, Brinkhues S (2014) A novel scheme for the approximation of residual stresses in arterial walls Archive of Applied Mechanics 84:881-898 doi:10.1007/s00419-014-0838-x

Sokolis DP (2015) Effects of aneurysm on the directional, regional, and layer distribution of residual strains in ascending thoracic aorta Journal of the Mechanical Behavior of Biomedical Materials 46:229-243 doi:http://dx.doi.org/10.1016/j.jmbbm.2015.01.024

Sokolis DP, Kritharis EP, Iliopoulos DC (2012) Effect of layer heterogeneity on the biomechanical properties of ascending thoracic aortic aneurysms Medical \& Biological Engineering \& Computing 50:1227-1237 doi:10.1007/s11517-012-0949-x

Vaishnav RN, Vossoughi J (1983) Residual stress and strain in aortic segments Journal of Biomechanics 20:235-237 doi:10.1016/0021-9290(87)90290-9

Vande Geest JP, Di Martino ES, Bohra A, Makaroun MS, Vorp DA (2006) A Biomechanics-Based Rupture Potential Index for Abdominal Aortic Aneurysm Risk Assessment Annals of the New York Academy of Sciences 1085:11-21 doi:10.1196/annals.1383.046

Weisbecker H, Pierce DM, Holzapfel GA (2014) A generalized prestressing algorithm for finite element simulations of preloaded geometries with application to the aorta International Journal for Numerical Methods in Biomedical Engineering 30:857-872 doi:10.1002/cnm.2632

Weisbecker H, Pierce DM, Regitnig P, Holzapfel GA (2012) Layer-specific damage experiments and modeling of human thoracic and abdominal aortas with non-atherosclerotic intimal thickening Journal of the Mechanical Behavior of Biomedical Materials 12:93-106 doi:http://dx.doi.org/10.1016/j.jmbbm.2012.03.012 


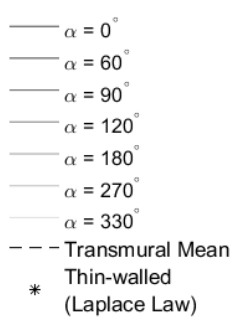

54

Figure 1 the transmural mean, thin-walled and thick-walled hoop stresses across the wall thickness. In the left figure, thick-walled hoop stresses were computed using Neo-Hookean model, while in

457 the right figure, GOH model was used. Transmural mean hoop stress remains the same for all 458 scenarios, thus only one line is plotted. 

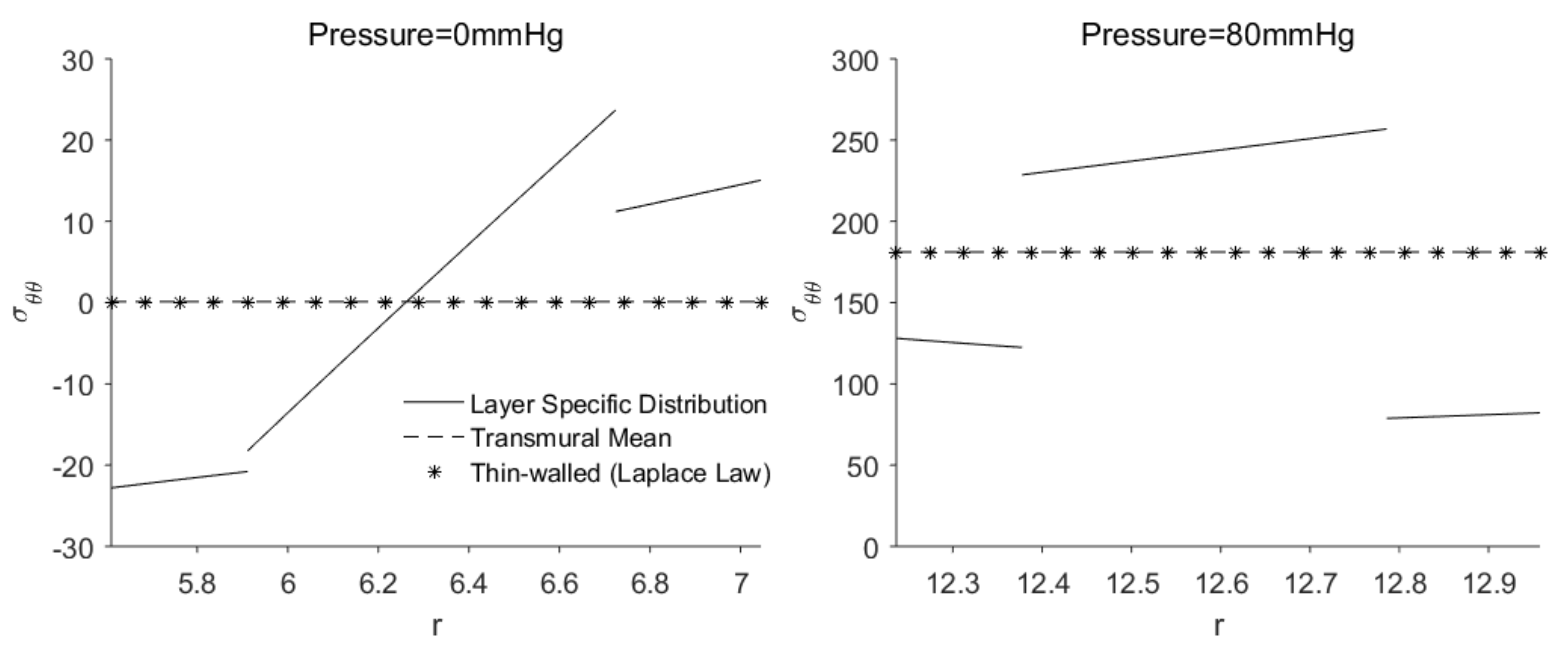

462 Figure 2 the transmural mean, thin-walled and layer-specific hoop stress distributions in the three

463 layer composite wall when 0 and 80 mmHg pressures are applied. 

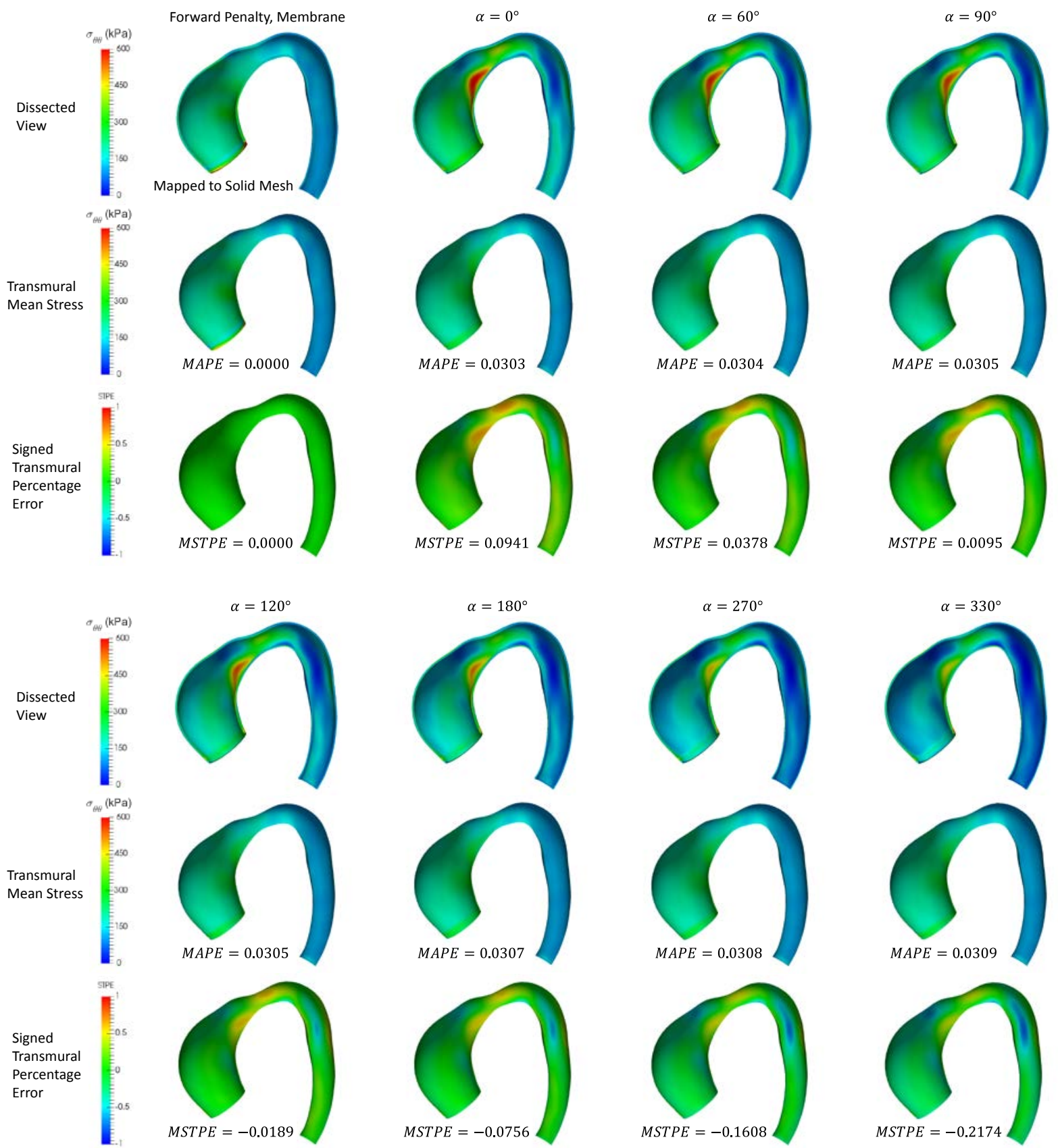

Figure 3 predicted results using the forward penalty approach and the GPA approach with different opening angles: (1) the hoop stress distribution in the dissected view (row 1 and row 4), (2) the transmural mean hoop stress (row 2 and row 5), and (3) the signed transmural percentage error (row 3 and row 6). 
471
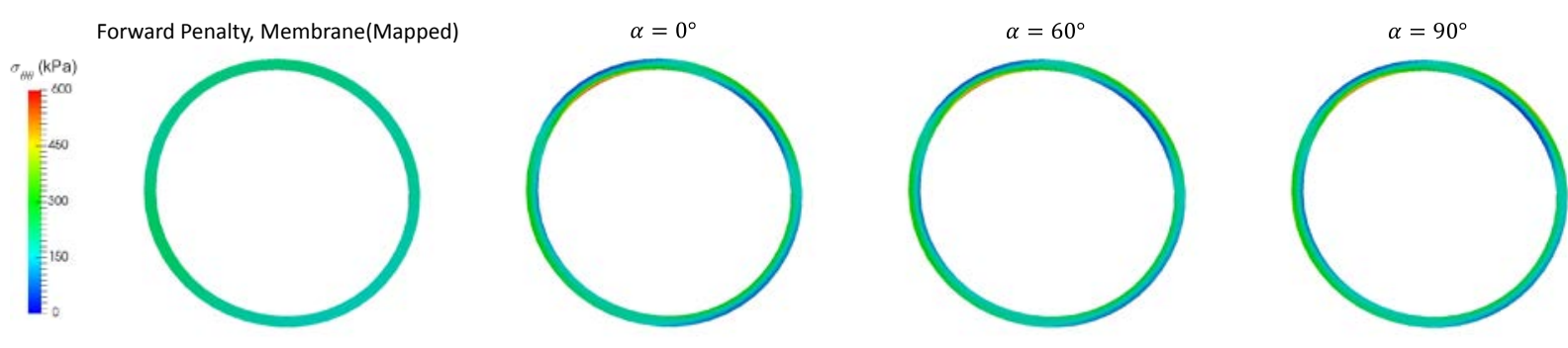

472
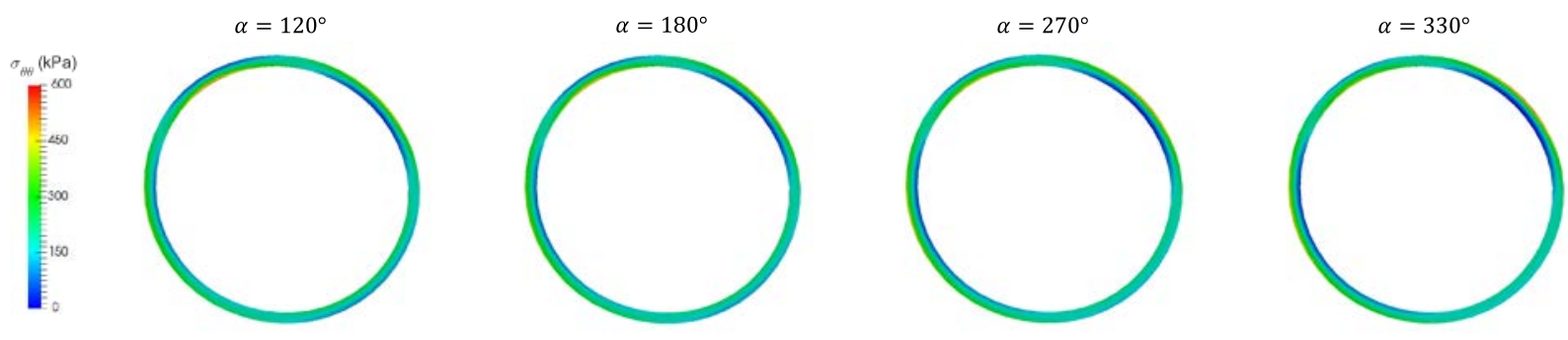

473 Figure 4 hoop stress in aortic rings using the forward penalty approach and the iterative approach

474 (GPA) with different opening angles. 

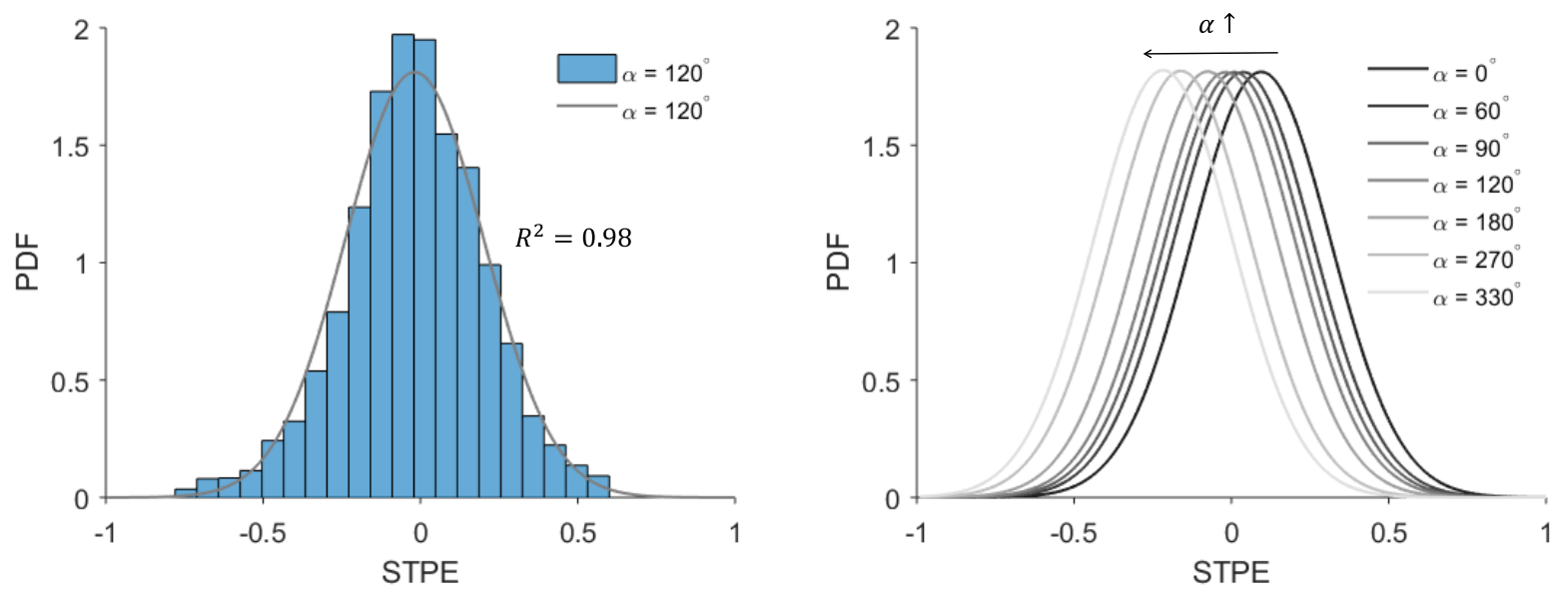

478 Figure 5 the probability density function (PDF) of the STPE is shown in the histogram and fitted

479 using the Gaussian distribution (left) and fitted PDFs correspond to different opening angles (right). 


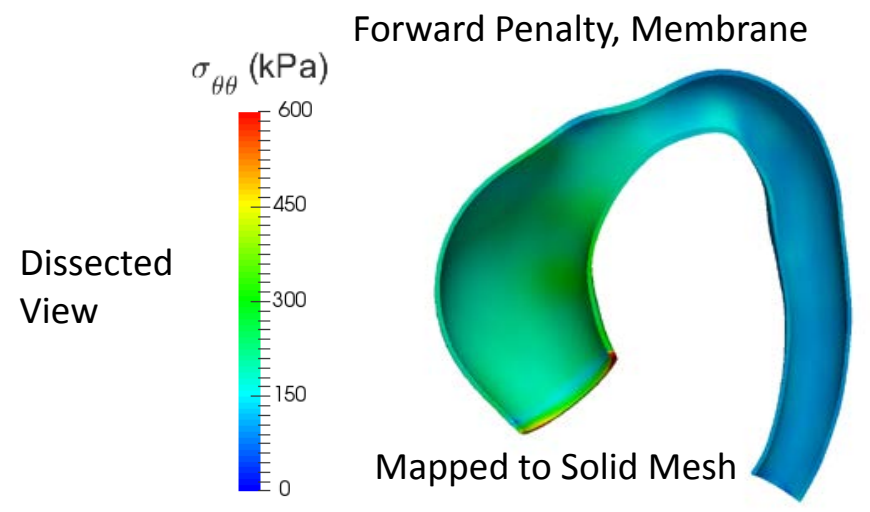

Layer Specific 3D Residual Deformation
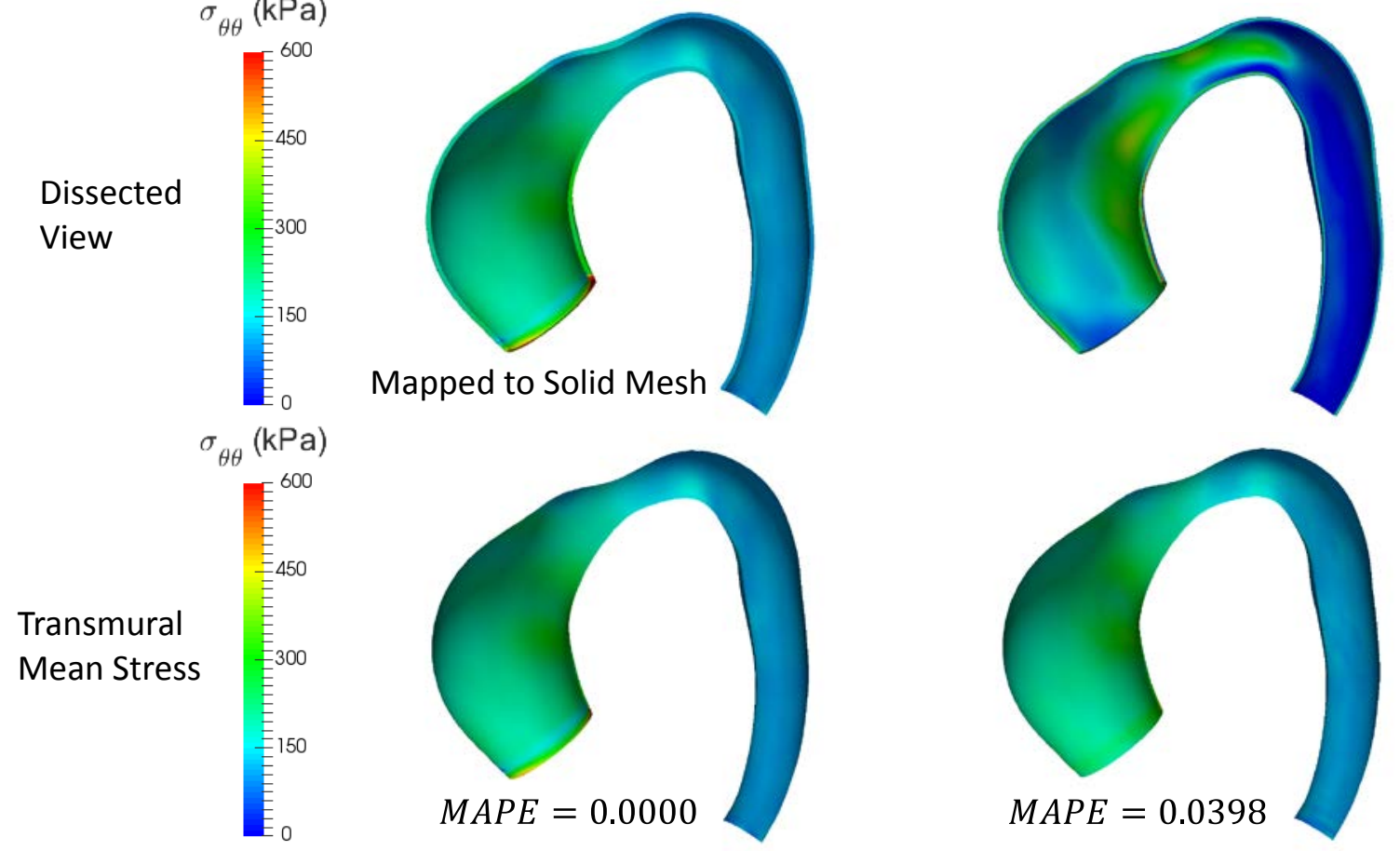

483 Figure 6 predicted results using the forward penalty approach and the iterative approach (GPA)

484 with layer-specific three-dimensional residual deformations: (1) the hoop stress distribution in the 485 dissected view (row 1), (2) the transmural mean hoop stress (row 2). 
Forward Penalty, Membrane(Mapped)
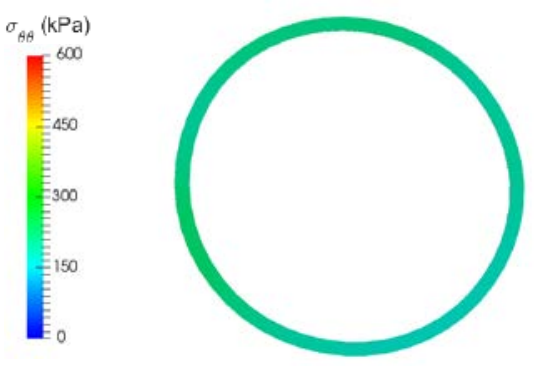
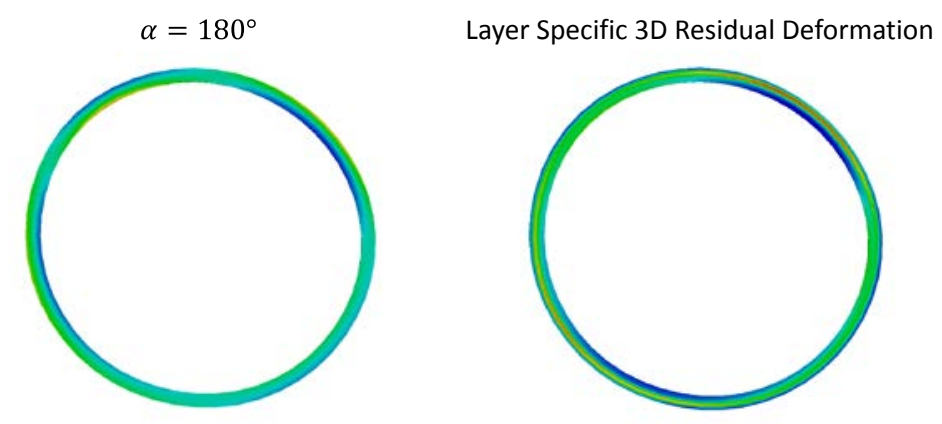

489

Figure 7 the hoop stress distribution in the aortic rings using the forward approach, the opening

490 angle method ( $\alpha=180^{\circ}$ ) and the layer-specific 3D residual deformation.

491 


\begin{tabular}{|l|l|l|l|l|}
\hline inner radius $a$ & outer radius $b$ & residual axial stretch & systolic pressure & shear modulus $\mu$ \\
$(\mathrm{mm})$ & $(\mathrm{mm})$ & $l / L$ & $(\mathrm{mPa})$ \\
\hline $24.5^{\mathrm{a}}$ & $26^{\mathrm{b}}$ & $1.2^{\mathrm{c}}$ & $104^{\mathrm{a}}$ & $67.68^{\mathrm{a}}$ \\
\hline
\end{tabular}

${ }^{a}$ from (Martin et al. 2015), $a$ and systolic pressure are from clinical recorded data, $\mu$ was fitted

494 using biaxial experiment of patient "BAV17" with coefficient of determination of 0.8656 ; ${ }^{\text {b }}$ based

495 on mean value of deformed wall thickness in (Liang et al. 2017); ${ }^{\mathrm{c}}$ approximated from (Guo and

496 Kassab 2003) which refers to the residual axial stretch. We assume there is no axial tension caused

497 by in vivo loading conditions

498 Table 1 the parameters used in the opening angle method. 


\begin{tabular}{|c|c|c|c|c|c|c|c|}
\hline \multicolumn{8}{|c|}{ Neo-Hookean Model } \\
\hline$\alpha\left(^{\circ}\right)$ & 0 & 60 & 90 & 120 & 180 & 270 & 330 \\
\hline$A(m m)$ & 11.55 & 14.20 & 15.96 & 18.16 & 24.77 & 51.23 & 157.10 \\
\hline$B(m m)$ & 14.98 & 17.62 & 19.39 & 21.59 & 28.20 & 54.67 & 160.54 \\
\hline \multicolumn{8}{|c|}{ Gasser-Ogden-Holzapfel (GOH) Model } \\
\hline$\alpha\left(^{\circ}\right)$ & 0 & 60 & 90 & 120 & 180 & 270 & 330 \\
\hline$A(m m)$ & 18.25 & 22.12 & 24.70 & 27.93 & 37.63 & 76.43 & 231.66 \\
\hline$B(m m)$ & 20.59 & 24.46 & 27.04 & 30.26 & 39.97 & 78.77 & 234.00 \\
\hline
\end{tabular}

500 Table 2 the inner and outer radii $A$ and $B$ of the stress-free configurations corresponding to

501 various opening angles $\alpha$. 


\begin{tabular}{|c|c|c|c|c|}
\hline $\mathrm{C}_{10}(k P a)$ & $\mathrm{k}_{1}(k P a)$ & $\mathrm{k}_{2}$ & $\kappa$ & $\theta\left(^{\circ}\right)$ \\
\hline 27.91 & 512.56 & 0.00 & 0.31 & 90.00 \\
\hline
\end{tabular}

503 Table $3 \mathrm{GOH}$ material parameters of the patient "BAV17” extracted from (Martin et al. 2015).

504 Coefficient of determination of the curve fitting is 0.9551. 


\begin{tabular}{|c|c|c|}
\hline Intima & Media & Adventitia \\
\hline$A^{(I)}=7.50 m m$ & $A^{(M)}=8.41 \mathrm{~mm}$ & $L_{1}^{(A)}=0.21 \mathrm{~mm}$ \\
$B^{(I)}=7.76 \mathrm{~mm}$ & $B^{(M)}=8.99 \mathrm{~mm}$ & $L_{2}^{(A)}=18.35 \mathrm{~mm}$ \\
$L^{(I)}=2.58 \mathrm{~mm}$ & $L^{(M)}=2.52 \mathrm{~mm}$ & $L_{3}^{(A)}=2.29 \mathrm{~mm}$ \\
$k^{(I)}=1.19$ & $k^{(M)}=2.79$ & $b^{(A)}=7.05 \mathrm{~mm}$ \\
$a^{(I)}=5.61 \mathrm{~mm}$ & $\mu^{(M)}=31.4 \mathrm{kPa}$ & $\mu^{(A)}=17.3 \mathrm{kPa}$ \\
$\mu^{(I)}=39.8 \mathrm{kPa}$ & & \\
\hline
\end{tabular}

506 Table 4 material and residual deformation parameters from (Holzapfel and Ogden 2010;

507 Holzapfel et al. 2007). In addition, $l=2.48 m m, b^{(I)}=a^{(M)}$ and $b^{(M)}=a^{(A)}$ can be calculated 508 according to (Holzapfel and Ogden 2010). 


\begin{tabular}{|c|c|c|c|}
\hline Wall thickness (mm) & 1.0 & 2.0 & 3.0 \\
\hline MAPE & 0.0191 & 0.0355 & 0.0458 \\
\hline
\end{tabular}




\begin{tabular}{|c|c|c|c|c|c|}
\hline & $\mathrm{C}_{10}(k P a)$ & $\mathrm{k}_{1}(k P a)$ & $\mathrm{k}_{2}$ & $\kappa$ & $\theta\left(^{\circ}\right)$ \\
\hline Intima & 17 & 4340 & 13.32 & 0.20 & 46.5 \\
\hline Media & 14 & 140 & 11.90 & 0.21 & 38.4 \\
\hline Adventitia & 10 & 390 & 6.79 & 0.23 & 52.3 \\
\hline
\end{tabular}

513 Table 6 layer-specific GOH material parameters from (Weisbecker et al. 2012).

514

515

516

517

518

519

520

521

522

523

524 